\title{
HACIA EL MODELADO DE MATERIAL PARTICULADO FINO EN SANTIAGO, CHILE, MEDIANTE IMÁGENES MODIS
}

\section{TOWARD THE MODELING OF FINE PARTICULATE MATTER IN SANTIAGO, CHILE, BY MODIS IMAGES}

\author{
Sr. M.A. Peña ${ }^{1}$ y Sr. Pablo Araya ${ }^{2}$
}

\section{RESUMEN}

Este estudio exploró la capacidad que ofrece el producto de profundidad óptica de aerosoles (aerosol optical depth, AOD) creado diariamente desde imágenes MODIS (Moderate Resolution Imaging Spectroradiometer) adquiridas a las 10:30 AM y 1:30 PM hora local, para predecir las concentraciones superficiales de material particulado fino $\left(\mathrm{MP}_{2,5}\right)$, medidas en las estaciones de monitoreo de la ciudad de Santiago, Chile, durante los días libres de nubosidad del año 2016. Para esto, fueron realizadas correlaciones lineales entre ambas fuentes de datos, remotos y de terreno, calzados en tiempo y espacio, que permitieron dilucidar los días y estaciones donde MODIS-AOD puede ofrecer la mayor correlación. Considerando estos casos fue realizado un modelo de regresión lineal simple que permitió explorar la capacidad predictiva de MODIS-AOD. Las mayores correlaciones fueron consistentemente obtenidas cuando los datos MODIS-AOD creados a la 1:30 PM fueron considerados por estación a lo largo de todos los días muestreados en el período de interés. Al agrupar las estaciones de Cerro Navia, Quilicura y Pudahuel, el coeficiente de correlación fue 0,77 , en tanto que el modelo de regresión explicó el $63 \%$ del comportamiento de las concentraciones superficiales de $\mathrm{MP}_{2,5}$ y mostró una precisión de $69,1 \%$. Los hallazgos de este estudio exploratorio constituyen una primera aproximación al modelado de las concentraciones superficiales de $\mathrm{MP}_{2,5}$ en la ciudad de Santiago, con miras a ser robustecido a futuro, para con ello contribuir a prever alertas ambientales y ocurrencias de enfermedades relacionadas a la exposición del contaminante.

Palabras clave: MODIS, material particulado fino, regresión lineal, serie temporal de imágenes.

\section{ABSTRACT}

This study explored the capabilities offered by the aerosol optical depth (AOD) product (created on a daily basis from MODIS (Moderate Resolution Imaging Spectroradiometer) images acquired at 10:30 AM and 1:30 PM local time, to predict the at-surface concentrations of fine particulate matter ( $\left.\mathrm{PM}_{2.5}\right)$ measured on the monitoring stations of the city of Santiago, Chile, during free cloud days of year 2016. To accomplish that, linear correlations were performed between remote-based and field-based data sources, both matched in time and space, in order to find the days and stations where MODIS-AOD may offer the highest correlation. Based on these cases, a linear regression model was performed in order to explore the predictive power of MODIS-AOD. The highest correlations were consistently obtained when MODIS-AOD data created at 1:30 PM were used at the station level for all the sampled days of the study period. When the monitoring stations of Cerro Navia, Quilicura and Pudahuel were grouped, the correlation coefficient was 0.77 and the predictive model explained the $63 \%$ of the at-surface $\mathrm{MP}_{2.5}$ concentrations and yielded an accuracy of $69.1 \%$. The findings of this exploratory study provide a first approach to the modeling of the at-surface concentrations of fine particulate matter in the city of Santiago, which could be improved in a future with the aim to anticipate environmental warnings and disease outbreaks related to the exposure of this pollutant.

Keywords: MODIS, fine particulate matter, linear regression, image time series.

\footnotetext{
${ }^{1}$ Departamento de Geografía, Universidad Alberto Hurtado, Cienfuegos 41, Santiago, Chile.

2 Departamento de Geografía, Universidad Alberto Hurtado, Cienfuegos 41, Santiago, Chile.
}

Fecha de Recepción: 15 de julio de 2019

Fecha de Aprobación: 22 de diciembre de 2019 


\section{INTRODUCCIÓN}

Durante las últimas décadas ha sido ampliamente evidenciado el efecto negativo que tiene en la salud humana la exposición prolongada a la polución del aire por material particulado fino de carácter antropogénico (diámetro aerodinámico $\leq 2,5 \mu \mathrm{m}$, en adelante $\mathrm{MP}_{2,5}$ ). Este contaminante es una mezcla física y químicamente heterogénea de partículas sólidas y líquidas que por su pequeño tamaño permanecen suspendidas en el aire, ocasionando subsecuentemente, enfermedades cardiopulmonares y muertes prematuras (ver Kim et al., 2015, para más detalles y referencias). Para valorar los efectos del $\mathrm{MP}_{2,5}$ sobre la salud humana, son necesarias mediciones espacial $y$ temporalmente regulares de sus concentraciones a nivel superficial. Sin embargo, debido a la distribución espacial típicamente dispersa de estas mediciones, tal valoración depende fuertemente de modelos de interpolación espacial que introducen incertidumbres (Liu et al., 2007; Evans et al., 2014). El sensor óptico satelital MODIS (Moderate Resolution Imaging Spectroradiometer), operativo desde mediados de 2000, provee datos espacial y temporalmente continuos de la carga de aerosoles atmosféricos, usados como proxies para estimar concentraciones superficiales de $\mathrm{MP}_{2,5}$. Específicamente el producto de grosor 0 profundidad óptica de aerosoles (aerosol optical thickness/depth, AOT/AOD) mide la radiancia atenuada o extinta por el esparcimiento y absorción de aerosoles contenidos en la columna total de la atmósfera que yace sobre el terreno muestreado por el sensor. Para esto, relaciona modelos de aerosoles preestablecidos con bandas de la imagen MODIS que permiten aislar su reflectancia en la columna atmosférica (ver detalles en Liang et al., 2006). Diariamente dos productos MODIS-AOD son generados, uno adquirido desde el satélite Terra a las 10:30 AM hora local aproximadamente (MOD04), y otro adquirido desde el satélite Aqua a la 1:30 PM hora local aproximadamente (MYD04), ambos espacialmente remuestreados a un píxel de $10 \mathrm{~km}$ con el sentido de aumentar la razón señalruido.

Muchos estudios han encontrado claras correlaciones lineales positivas entre MODIS-AOD $y$ las concentraciones superficiales de MP, especialmente aquellas más finas, debido a la sensibilidad de las longitudes de onda óptica o solar a ser esparcidas o absorbidas por partículas de diámetro similar a la anchura de éstas (Wang y Christopher, 2003; Kumar et al., 2007; Tsai et al.,
2008). Lo anterior ha permitido establecer modelos predictivos sobre el comportamiento del contaminante a nivel local (Hutchison et al., 2004; Liu et al., 2007), regional (Engel-Cox et al., 2004; Van Donkeelar et al., 2006) y global (Gupta et al., 2006; Van Donkeelar et al., 2010), así como sobre la ocurrencia de enfermedades y mortalidades asociadas a éste (Hu, 2009; Evans et al., 2013).

Estas correlaciones ocurren a pesar de las diferentes resoluciones que presentan ambas fuentes de datos. Mientras las mediciones de $\mathrm{MP}_{2,5}$ realizadas mediante estaciones de monitoreo son de carácter puntual, las mediciones de AOD derivadas de MODIS abarcan el área de un píxel. Por otro lado, los datos de $\mathrm{MP}_{2,5}$ resultan usualmente de un promedio horario, en tanto que Ios datos AOD son generados a partir del momento de adquisición de la imagen MODIS. Finalmente, mientras los datos de $\mathrm{MP}_{2,5}$ representan la concentración de contaminantes a nivel de la capa atmosférica en contacto con la superficie, los datos MODIS-AOD entregan una medida indirecta (y adimensional) acerca de su contenido a lo largo de toda la columna atmosférica interpuesta entre el sensor y la superficie que observa.

El presente trabajo examina la relación entre las concentraciones superficiales de $\mathrm{MP}_{2,5}$ y MODISAOD en la ciudad de Santiago, Chile, donde no existen estudios previos a este respecto, a pesar de la intensidad y persistencia que exhibe la contaminación atmosférica por MP y de la escasez de estaciones que lo monitorean (Willumsen, 2010). Tomando los casos que presentan las mayores correlaciones, el trabajo luego propone un modelo lineal para la predicción de las concentraciones superficiales del contaminante en cuestión. Este estudio piloto busca dar un primer paso en la cimentación de un modelo predictivo más complejo de concentración superficial de $\mathrm{MP}_{2,5}$ en la ciudad de Santiago, capaz de anticipar con suficiente precisión alertas ambientales y brotes de enfermedades asociados a éstas.

\section{MATERIALES Y MÉTODOS}

\section{ÁREA DE ESTUDIO}

El área de estudio corresponde a la ciudad de

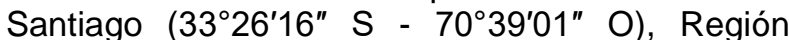
Metropolitana de Chile, que alberga una población de más de 5 millones de habitantes a lo largo de una superficie de $513,3 \mathrm{~km}^{2}$, aproximadamente. La Región se divide en seis provincias y éstas a su vez, en 52 comunas. El clima imperante es 
mediterráneo continental, caracterizado por lluvias invernales y una estación seca prolongada que se extiende desde fines de Septiembre hasta fines de Marzo, con temperaturas promedio de alrededor de $20^{\circ} \mathrm{C}$ y máximas que suelen sobrepasar los $30^{\circ} \mathrm{C}$ (SEGPRES, 2010).

\section{DESCARGA Y PREPARACIÓN DE DATOS DE AOD}

El producto MODIS-AOD, correspondiente al año 2016 del área de estudio, fue descargado gratuitamente desde el servicio en línea de la NASA (National Aeronautics and Space Administartion)https://search.earthdata.nasa.gov/s earch. Con el sentido de comparar los rendimientos de MODIS-AOD a diferentes horas del día, tanto los productos MOD04 como MYD04 fueron empleados. En ambos casos las descargas de imágenes fueron realizadas con una frecuencia de 10 días a partir del inicio del período de interés (1 de Enero de 2016) y hasta el final de éste (31 de Diciembre de 2016), interrumpiéndola en los meses comprendidos entre Mayo y Septiembre, a causa de la prevalecencia de nubosidad. De forma similar, debido a la pérdida de datos por nubosidad estival principalmente, varias de las fechas predefinidas por el intervalo de descarga debieron ser reajustadas al día más próximo libre de nubosidad. El mismo intervalo fue empleado para la descarga de MYD04, pero intercalado entre aquel definido para MOD04, comenzando desde el 15 de Enero de 2016 (ya que el 5 de Enero no presentó datos para el área de estudio).

Como resultado, 31 imágenes MODIS-AOD fueron finalmente empleadas para el período de interés: 19 adquiridas por Terra y 12 adquiridas por Aqua. Éstas fueron leídas y preparadas en el programa ENVI (Environment for Visualizing Images) 5.3, con el propósito de extraer los valores de los píxeles coincidentes espacialmente con cada una de las 10 estaciones de monitoreo emplazadas al interior de la ciudad de Santiago (ver próximo acápite).

\section{DESCARGA Y PREPARACIÓN DE DATOS DE $\mathrm{MP}_{2,5}$}

Las concentraciones superficiales de $\mathrm{MP}_{2,5}$, correspondientes al año 2016 del área de estudio, fueron descargadas gratuitamente desde el servicio en línea del Sistema de Información Nacional de Calidad del Aire (SINCA) http://sinca.mma.gob.cl/. Estos datos son provistos a nivel horario para la Red de Monitoreo Automático de Calidad del Aire de la Región Metropolitana (MACAM), que en el área de estudio consta de 10 estaciones ubicadas en las comunas de Cerrillos, Cerro Navia, El Bosque, Independencia, La Florida, Las Condes, Pudahuel, Puente Alto, Quilicura y Santiago (designada Parque O'Higgins), todas inscritas en la provincia de Santiago (Figura 1). Específicamente, fueron descargadas aquellas mediciones efectuadas a las horas más cercanas a la adquisición de las imágenes MODIS-AOD previamente descargadas. Estos datos fueron empleados tanto corregidos como no corregidos por humedad atmosférica. Para lo primero, fue aplicada la fórmula referida en Tsai et al., (2009):

$$
A O D_{H R}=A O D \times(1 /(1-H R / 100))
$$

Donde, $A O D_{H R}$ es el dato MODIS AOD corregido por humedad relativa y HR es la humedad relativa medida para ese punto espacial. Esta corrección tuvo el propósito de aproximar las mediciones de $\mathrm{MP}_{2,5}$ realizadas bajo condiciones de humedad controlada en cada estación, a aquellas imperantes en el ambiente en ese momento.

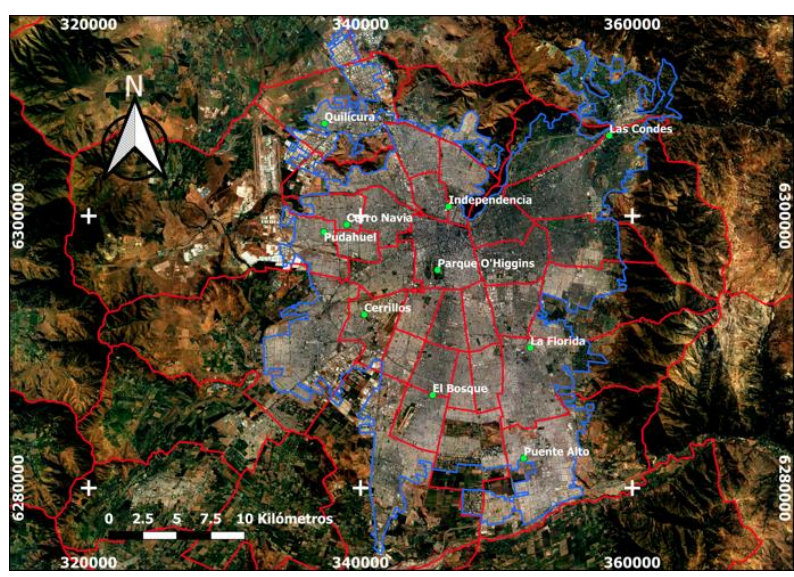

Figura 1. Ubicación de las estaciones de monitoreo de calidad del aire en la ciudad de Santiago. Las líneas rojas representan límites comunales y la línea azul representa el límite de la superficie urbanizada de Santiago. Imagen satelital: cortesía de GoogleEarth@.

\section{RELACIÓN ESTADÍSTICA ENTRE DATOS REMOTOS Y DE TERRENO}

Los datos remotos y de terreno fueron sujetos a correlaciones de Pearson con el sentido de seleccionar aquellos casos con los mayores coeficientes de correlación y por lo tanto, con la mayor potencialidad para su posterior modelado 
predictivo. Estas correlaciones fueron efectuadas en términos temporales y espaciales. Es decir, en el primer caso fueron realizadas correlaciones entre las concentraciones de $\mathrm{MP}_{2,5}$ medidas en todas las estaciones y sus respectivos datos MODIS-AOD según día del período de interés. En el segundo caso, fueron realizadas correlaciones entre las concentraciones de $\mathrm{MP}_{2,5}$ medidas en cada estación y sus respectivos datos MODIS-AOD a lo largo de todos los días del período de interés, separándolas además según el satélite de captura. A causa de la pérdida de algunos datos remotos 0 de terreno, las correlaciones consideraron diferentes números de pares de datos disponibles, lo cual fue contabilizado a fin de tener una idea acerca de la muestra finalmente válida en cada caso.

\section{MODELADO DE CONCENTRACIONES SUPERFICIALES DE MP 2,5}

En atención a las correlaciones obtenidas en el paso anterior, fueron seleccionados sólo los casos que arrojaron las mayores correlaciones, con el propósito de explorar el rendimiento de un modelo de regresión lineal simple sólo para éstos. En este punto fue examinado el coeficiente de determinación $\left(\mathrm{R}^{2}\right)$ arrojado por el modelo, así como la raíz del error cuadrático medio (root mean square error, RMSE) resultante de los valores de $\mathrm{MP}_{2,5}$ reales y aquellos predichos por éste mediante validación cruzada, a partir de lo cual fue obtenida la precisión predictiva del modelo.

\section{RESULTADOS}

\section{CORRELACIÓN ENTRE DATOS REMOTOS Y DE TERRENO SEGÚN DÍA}

Los coeficientes de correlación entre la serie completa de datos MODIS-AOD y las concentraciones superficiales de $\mathrm{MP}_{2,5}$ según día de interés, considerando las 10 estaciones de monitoreo ( Error! No se encuentra el origen de la referencia.), fueron bajos e incluso negativos, independiente del tipo de producto empleado.

Tabla 1. Coeficientes de correlación ( $r$ ) entre MODIS$A O D$ y las concentraciones superficiales de $M_{2,5}$ según día para todas las estaciones de monitoreo. Fuente: Elaboración propia.

\begin{tabular}{lll}
\hline \multirow{2}{*}{$\#$} & \multirow{2}{*}{ Día } & $\mathrm{MP}_{2,5}$ \\
\cline { 2 - 3 } & Crudo & Corregido \\
\hline
\end{tabular}

\begin{tabular}{|c|c|c|c|c|c|c|}
\hline & & $r$ & 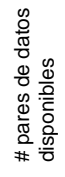 & $r$ & 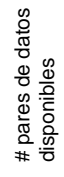 & $\begin{array}{c}\text { Producto } \\
\text { MODIS- } \\
\text { AOD }\end{array}$ \\
\hline 1 & 1 de Enero & $-0,66$ & 8 & $-0,64$ & 7 & MOD04 \\
\hline 2 & 11 de Enero & $-0,15$ & 9 & $-0,13$ & 8 & MOD04 \\
\hline 3 & 15 de Enero & 0,09 & 9 & $-0,41$ & 7 & MYD04 \\
\hline 4 & 21 de Enero & $-0,34$ & 8 & $-0,02$ & 7 & MOD04 \\
\hline 5 & 31 de Enero & $-0,18$ & 9 & $-0,13$ & 8 & MOD04 \\
\hline 6 & 5 de Febrero & $-0,52$ & 8 & - & 1 & MYD04 \\
\hline 7 & 10 de Febrero & $-0,08$ & 9 & 0,04 & 9 & MOD04 \\
\hline 8 & 15 de Febrero & $-0,13$ & 8 & - & 1 & MYD04 \\
\hline 9 & 17 de Febrero & $-0,66$ & 6 & $-0,1$ & 6 & MOD04 \\
\hline 10 & 25 de Febrero & 0,26 & 4 & 0,5 & 4 & MYD04 \\
\hline 11 & 28 de Febrero & 0,74 & 6 & 0,71 & 6 & MOD04 \\
\hline 12 & 1 de Marzo & 0,07 & 7 & 0,05 & 7 & MOD04 \\
\hline 13 & 10 de Marzo & $-0,11$ & 8 & $-0,3$ & 8 & MOD04 \\
\hline 14 & 21 de Marzo & $-0,22$ & 9 & $-0,03$ & 9 & MOD04 \\
\hline 15 & 25 de Abril & - & 0 & - & 0 & MYD04 \\
\hline 16 & 30 de Abril & 0,02 & 8 & 0,12 & 8 & MOD04 \\
\hline 17 & 1 de Septiembre & $-0,47$ & 7 & $-0,28$ & 6 & MOD04 \\
\hline 18 & 21 de Septiembre & 0,17 & 10 & 0,09 & 8 & MOD04 \\
\hline 19 & 25 de Septiembre & 0,39 & 10 & 0,62 & 9 & MYD04 \\
\hline 20 & 5 de Octubre & $-0,24$ & 10 & 0,21 & 9 & MYD04 \\
\hline 21 & 15 de Octubre & - & 0 & - & 0 & MYD04 \\
\hline 22 & 21 de Octubre & $-0,33$ & 5 & $-0,54$ & 4 & MOD04 \\
\hline 23 & 25 de Octubre & 0,52 & 6 & 0,46 & 5 & MYD04 \\
\hline 24 & 4 de Noviembre & 0,17 & 10 & $-0,44$ & 8 & MYD04 \\
\hline 25 & 10 de Noviembre & 0,28 & 10 & $-0,34$ & 8 & MOD04 \\
\hline 26 & 14 de Noviembre & 0,48 & 10 & 0,11 & 8 & MYD04 \\
\hline 27 & 30 de Noviembre & $-0,49$ & 7 & 0,2 & 6 & MOD04 \\
\hline 28 & 4 de Diciembre & 0,11 & 3 & - & 1 & MYD04 \\
\hline 29 & 10 de Diciembre & - & 2 & - & 2 & MOD04 \\
\hline 30 & 14 de Diciembre & $-0,44$ & 10 & - & 1 & MYD04 \\
\hline 31 & 20 de Diciembre & $-0,05$ & 9 & $-0,08$ & 7 & MOD04 \\
\hline 32 & 24 de Diciembre & $-0,57$ & 10 & $-0,49$ & 7 & MYD04 \\
\hline \multirow[t]{2}{*}{33} & 30 de Diciembre & 0,28 & 10 & 0,39 & 8 & MOD04 \\
\hline & Todos & 0,08 & 245 & 0,11 & 193 & $\begin{array}{l}\text { MOD04/ } \\
\text { MYD04 }\end{array}$ \\
\hline
\end{tabular}

\section{CORRELACIÓN ENTRE DATOS REMOTOS Y DE TERRENO SEGÚN ESTACIÓN}

Las correlaciones obtenidas según estación de monitoreo para todos los días del período de estudio (Tabla 2), muestran una tendencia similar a la anteriormente descrita, sin mostrar un incremento apreciable al aplicar sobre los datos de terreno la corrección por humedad relativa.

\begin{tabular}{|c|c|c|c|c|}
\hline \multirow[b]{3}{*}{ Estación } & \multicolumn{4}{|c|}{$\mathrm{MP}_{2,5}$} \\
\hline & \multicolumn{2}{|c|}{ Crudo } & \multicolumn{2}{|c|}{ Corregido } \\
\hline & $r$ & $\begin{array}{c}\text { \# pares de } \\
\text { datos } \\
\text { disponibles }\end{array}$ & $r$ & $\begin{array}{c}\text { \# pares de } \\
\text { datos } \\
\text { disponibles }\end{array}$ \\
\hline Puente alto & 0,25 & 27 & 0,03 & 16 \\
\hline Cerrillos & $-0,18$ & 26 & $-0,13$ & 25 \\
\hline Cerro Navia & 0,05 & 27 & $-0,18$ & 24 \\
\hline El Bosque & $-0,18$ & 27 & $-0,18$ & 25 \\
\hline Independencia & $-0,21$ & 26 & $-0,2$ & 24 \\
\hline La Florida & 0,3 & 25 & 0,29 & 22 \\
\hline Las Condes & 0,27 & 23 & 0,4 & 15 \\
\hline Pudahuel & $-0,13$ & 25 & $-0,11$ & 22 \\
\hline Quilicura & 0,62 & 15 & - & 0 \\
\hline Parque O'Higgins & $-0,06$ & 30 & $-0,11$ & 27 \\
\hline Todas & -011 & 251 & $-0,12$ & 200 \\
\hline
\end{tabular}

Tabla 2. Coeficientes de correlación ( $r$ ) entre MODISAOD y las concentraciones superficiales de $M_{2,5}$ según estación de monitoreo para todos los días del período de estudio. Fuente: Elaboración propia. 
Sin embargo, cuando los coeficientes de correlación fueron nuevamente obtenidos a nivel de estación, pero esta vez separando el tipo producto (Tabla 3 para MOD04 y Tabla 4 para MYD04), se revelan valores consistentemente positivos $y$ claramente más altos para el caso de MYD04. Asimismo, se puede apreciar que en gran parte de las estaciones los coeficientes de correlación aumentaron al aplicarse sobre los datos remotos la corrección por humedad relativa.

\begin{tabular}{|c|c|c|c|c|}
\hline \multirow[b]{3}{*}{ Estación } & \multicolumn{4}{|c|}{$\mathrm{MP}_{2,5}$} \\
\hline & \multicolumn{2}{|c|}{ Crudo } & \multicolumn{2}{|c|}{ Corregido } \\
\hline & $r$ & $\begin{array}{c}\text { \# pares de } \\
\text { datos } \\
\text { disponibles }\end{array}$ & $r$ & $\begin{array}{c}\text { \# pares de } \\
\text { datos } \\
\text { disponibles }\end{array}$ \\
\hline Puente alto & $-0,23$ & 17 & $-0,01$ & 13 \\
\hline Cerrillos & $-0,3$ & 16 & $-0,24$ & 17 \\
\hline Cerro Navia & $-0,25$ & 18 & $-0,23$ & 18 \\
\hline El Bosque & $-0,33$ & 16 & $-0,34$ & 17 \\
\hline Independencia & $-0,34$ & 16 & $-0,35$ & 17 \\
\hline La Florida & 0 & 14 & 0,17 & 14 \\
\hline Las Condes & 0,08 & 12 & 0,28 & 8 \\
\hline Pudahuel & $-0,25$ & 16 & $-0,2$ & 16 \\
\hline Quilicura & $-0,54$ & 7 & 2 & 0 \\
\hline Parque O'Higgins & $-0,18$ & 19 & $-0,24$ & 19 \\
\hline Todas & $-0,23$ & 151 & $-0,12$ & 139 \\
\hline
\end{tabular}

Tabla 3. Coeficientes de correlación ( $r$ ) entre MOD04 y las concentraciones superficiales de $\mathrm{MP}_{2,5}$ según estación de monitoreo para todos los días del período de estudio. Fuente: Elaboración propia.

\begin{tabular}{|c|c|c|c|c|}
\hline \multirow[b]{3}{*}{ Estación } & \multicolumn{4}{|c|}{$\mathrm{MP}_{2,5}$} \\
\hline & \multicolumn{2}{|c|}{ Crudo } & \multicolumn{2}{|c|}{ Corregido } \\
\hline & $r$ & $\begin{array}{c}\text { \# par de } \\
\text { datos } \\
\text { disponibles }\end{array}$ & $r$ & $\begin{array}{c}\text { \# par de } \\
\text { datos } \\
\text { disponibles }\end{array}$ \\
\hline Puente alto & 0,25 & 10 & $\begin{array}{l}-0,31 \\
\end{array}$ & 3 \\
\hline Cerrillos & 0,69 & 10 & 0,42 & 8 \\
\hline Cerro Navia & 0,75 & 9 & 0,85 & 6 \\
\hline El Bosque & 0,21 & 11 & 0,55 & 8 \\
\hline Independencia & 0,54 & 10 & 0,74 & 7 \\
\hline La Florida & 0,55 & 11 & 0,62 & 8 \\
\hline Las Condes & 0,58 & 11 & 0,74 & 7 \\
\hline Pudahuel & 0,52 & 9 & 0,71 & 6 \\
\hline Quilicura & 0,91 & 8 & - & 0 \\
\hline Parque O'Higgins & 0,55 & 11 & 0,65 & 8 \\
\hline Todas & 0,47 & 100 & 0,5 & 61 \\
\hline
\end{tabular}

Tabla 4. Coeficientes de correlación ( $r$ ) entre MYD04 y las concentraciones superficiales de $\mathrm{MP}_{2,5}$ según estación de monitoreo para todos los días del período de estudio. Fuente: Elaboración propia.

\section{MODELO PREDICTIVO DE CONCENTRACIONES SUPERFICIALES DE MP ${ }_{2,5}$}

Considerando los coeficientes de correlación por estación arrojados por MYD04 y las concentraciones superficiales de $\mathrm{MP}_{2,5}$ corregidas por humedad relativa, fueron realizadas distintas agrupaciones de datos en atención a la vecindad de las estaciones, para luego probar si así las correlaciones aumentaban y con ello proponer un modelo predictivo espacialmente más integrado para el contaminante (datos no mostrados). Al respecto, al agrupar las estaciones ubicadas al noroeste de la ciudad de Santiago; Quilicura, Cerro Navia y Pudahuel, tradicionalmente consignado como un sector altamente contaminado por MP entre otros (Katz, 2006), el coeficiente de correlación entre datos remotos y de terreno fue de 0,77 , razón por la cual el modelo predictivo exploratorio fue realizado para dicha agrupación de datos y por lo tanto, para dicha porción del área de estudio.

La Tabla 5 muestra los principales resultados obtenidos de la aplicación del modelo de regresión lineal simple entre datos remotos y de terreno para las estaciones de Cerro Navia, Quilicura y Pudahuel agrupadas. Se puede observar que el $63 \%$ de la variabilidad en las concentraciones superficiales de $\mathrm{MP}_{2,5}$ fue explicada por MYD04 mediante una relación encontrada significativa. En tanto, el RMSE obtenido entre los datos predichos por el modelo y los reales fue $11,45 \mu \mathrm{g} / \mathrm{m}^{3}$, valor que se mantiene casi igual una vez que el modelo es sujeto a validación cruzada, lo que indica que el RMSE obtenido fue poco sensible a variaciones en el tamaño muestral. De lo anterior se desprende una precisión de predicción del modelo de $69,1 \%$, valor que resultó de considerar el RMSE de las concentraciones de $\mathrm{MP}_{2,5}$ resultantes de la validación cruzada en relación con la media de dichas concentraciones en la realidad para las estaciones de interés $\left(37 \mu \mathrm{g} / \mathrm{m}^{3}\right)$.

\begin{tabular}{llllll}
\hline \multirow{5}{*}{ Estaciones } & \multicolumn{5}{c}{ Resultados de la regresión lineal simple } \\
\cline { 2 - 5 } & $\begin{array}{l}p \text { - } \\
\text { valor }\end{array}$ & $\mathrm{R}^{2}$ & $\begin{array}{l}\text { RMSE } \\
\text { entre } \\
\text { valores } \\
\text { predichos y } \\
\text { reales } \\
\left(\mu \mathrm{g} / \mathrm{m}^{3}\right)\end{array}$ & $\begin{array}{l}\text { RMSE de la } \\
\text { validación } \\
\text { cruzada } \\
\left(\mu \mathrm{g} / \mathrm{m}^{3}\right)\end{array}$ & $\begin{array}{l}\text { Precisión } \\
\text { de } \\
\text { predicción } \\
(\%)\end{array}$ \\
\hline $\begin{array}{l}\text { Cerro } \\
\text { Navia, } \\
\begin{array}{l}\text { Pudahuel y } \\
\text { Quilicura }\end{array}\end{array}$ & $<0,05$ & 0,63 & 11,45 & 12,4 & 69,1 \\
\hline
\end{tabular}

Tabla 5. Principales resultados arrojados por el modelo de regresión lineal entre las concentraciones superficiales de $\mathrm{MP}_{2,5}$ corregidas por humedad relativa y MYD04 para las estaciones de Cerro Navia, Pudahuel y Quilicura agrupadas. Fuente: Elaboración propia.

\section{DISCUSIÓN Y CONCLUSIONES}

Las correlaciones bajas e incluso negativas, encontradas entre datos remotos y de terreno para la gran mayoría de estaciones de monitoreo a lo largo del período de interés, no concuerdan con lo reportado mayoritariamente en la literatura científica (Hutchison et al., 2004; Liu et al., 2007). Sin embargo, una vez que las correlaciones por 
estación fueron realizadas separadamente según la hora de adquisición de MODIS-AOD, se obtuvieron relativamente altas correlaciones positivas para MYD04. La explicación a este resultado podría estribar en las condiciones meteorológicas que imperan en el área de estudio durante los meses estivales (Smith y Romero, 2016). En efecto, como es posible derivar del trabajo de Tsai et al. (2011), la marcada estabilidad atmosférica prevaleciente a nivel superficial a lo largo de las primeras horas de la mañana a causa del enfriamiento radiativo, podría hacer que las mediciones de las concentraciones superficiales de $\mathrm{MP}_{2,5}$ no representen convenientemente a aquellas exhibidas en la totalidad de la columna atmosférica, correlacionándose pobremente con las mediciones provistas por MODIS-AOD en las primeras horas de la mañana. En cambio, una vez que la capa de estabilidad atmosférica se expande a causa del calentamiento radiativo, las concentraciones de $\mathrm{MP}_{2,5}$ podrían distribuirse más homogéneamente a lo largo de la atmósfera, correlacionándose más claramente con las mediciones inherentemente verticales efectuadas por MODIS-AOD hacia las primeras horas de la tarde. Esta situación es ilustrada en la Figura 2, que basándose en lo discutido por Tsai et al. (2011) compara la distribución hipotética de $\mathrm{MP}_{2,5}$ a lo largo de la columna atmosférica a las horas de paso local de ambos satélites.

Tsai et al. (2011) encontraron que MODIS-AOD se correlacionó más claramente con las concentraciones superficiales de $\mathrm{MP}_{2,5}$ durante estaciones del año en que se mezcla más homogéneamente la columna atmosférica (i.e., otoño), favoreciendo una mejor distribución vertical de $\mathrm{MP}_{2,5}$. Estos hallazgos concuerdan con lo anteriormente discutido, excepto que en nuestro caso tal diferencia en las correlaciones fue encontrada a nivel diurno en lugar de estacional. Por su parte, Engel-Cox et al. (2004) encontraron correlaciones negativas entre las concentraciones superficiales de $\mathrm{MP}_{2,5}$ y MODIS-AOD en la parte Oeste de Estados Unidos, señalando como una de las posibles causas el transporte en altura de contaminantes desde otras regiones y su consecuente alteración en la distribución homogénea de aerosoles a lo largo de la columna atmosférica. Lo anterior es coincidente con lo referido por Tsai et al. (2011), excepto que la alteración referida opera en altitud en lugar de a nivel superficial, contribuyendo a reforzar la explicación de los resultados obtenidos en nuestro trabajo.

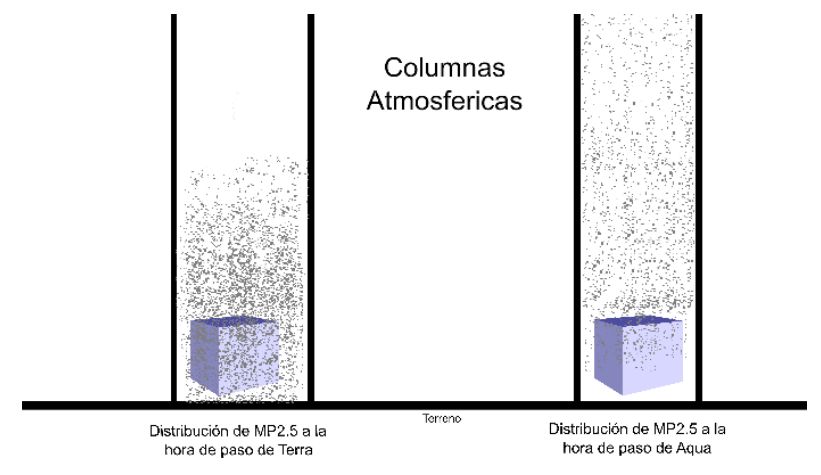

Figura 2. Concentración hipotética de $\mathrm{MP}_{2,5}$ en la columna atmosférica a las horas de paso local de los satélites Terra (10:30 AM) y Aqua (1:30 PM). Fuente: Elaboración propia.

Las correlaciones relativamente altas arrojadas por las estaciones de Cerro Navia, Quilicura y Pudahuel por separado y agrupadas sugieren una relativa homogeneidad en las concentraciones superficiales de $\mathrm{MP}_{2,5}$ de la columna atmosférica de esa área de la ciudad. Dicha área suele mostrar los peores índices de calidad del aire de la ciudad a lo largo del año, lo cual ha sido atribuido a la persistencia de condiciones de estabilidad asociadas al confinamiento de masas de aire sobre terrenos relativamente bajos (SEGPRES, 2010). Es probable que dicha estabilidad se mantenga en altura, explicando su particular relación con las mediciones de MYD04.

Es necesario destacar la limitación espacial que impone MODIS-AOD en el estudio de concentraciones de $\mathrm{MP}_{2,5}$ en el área de estudio, pues al tratarse de un producto de baja resolución espacial $(10 \mathrm{~km})$, el comportamiento espacial del contaminante se ve fuertemente agregado, impidiendo reconocer patrones espaciales a escala local e impactando en su correlación con datos puntuales derivados de estaciones de monitoreo. La Figura 3 ilustra la típica resolución espacial provista por MODIS-AOD sobre la ciudad de Santiago, pudiéndose apreciar que toda el área urbana es abarcada transversalmente por tres 0 cuatro píxeles. 


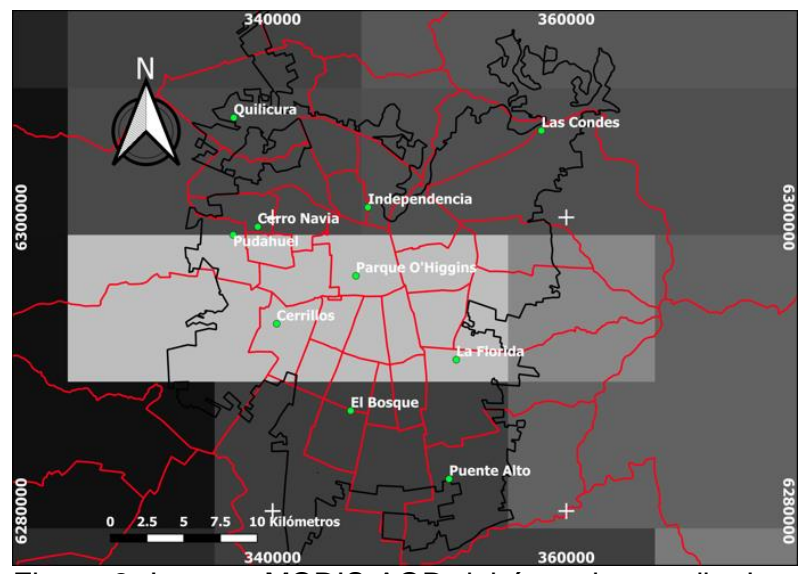

Figura 2. Imagen MODIS-AOD del área de estudio. Las líneas rojas representan límites comunales y la línea negra representa el límite de la superficie urbanizada de Santiago. Fuente: Elaboración propia.

Este estudio piloto consideró una muestra relativamente pequeña de datos a lo largo de días libres de nubosidad de un único año. Se espera que a futuro nuevos estudios amplíen tanto la muestra de datos como el período de interés, con el propósito de robustecer las correlaciones entre datos remotos y de terreno $y$, con ello, los resultados del modelo predictivo aquí explorado. En este sentido, es recomendable además corregir los datos de $\mathrm{MP}_{2,5}$ en función de la mezcla atmosférica esperada, de modo de aproximarlos a la naturaleza de las mediciones de MODIS-AOD. Asimismo, un estudio de este tipo podría ser ampliado a otras áreas del territorio nacional, teniendo presente las limitantes impuestas por la resolución espacial de MODIS-AOD, para así evaluar la posibilidad de proponer modelos predictivos espacialmente más integrados.

Los nuevos esfuerzos encausados en el modelado predictivo de las concentraciones superficiales de $\mathrm{MP}_{2,5}$ en el área de estudio mediante productos $A O D$, deberían incluir otras variables dependientes que han demostrado ser de ayuda a este respecto, como aquellas de tipo meteorológico y geográfico (Liu et al., 2005; Paciorek y Liu, 2009). También podrían ser considerados y contrastados otros productos AOD, como los derivados de los sensores satelitales MISR (Multiangle Imaging Spectroradiometer) y GOES (Geostationary Operational Environmental Satellite), que han demostrado ser útiles para tales fines (Liu et al., 2007; Paciorek y Liu, 2009; Evans et al., 2013). Asimismo, resultaría pertinente ampliar el período de estudio, valiéndose de la amplia disponibilidad temporal de datos horarios provistos por la Red MACAM para el área de estudio. Todo lo anterior permitiría contrastar los resultados aquí expuestos, a la vez de otorgarle mayor robustez al modelo propuesto.

De este estudio se desprende además, la necesidad de ahondar en las condiciones físicas diurnas y estacionales que presentan las principales capas atmosféricas del área de estudio, lo que contribuiría a profundizar en las explicaciones aquí esbozadas acerca de los resultados obtenidos, especialmente aquellos más promisorios concernientes a la hora de paso del satélite Aqua.

\section{REFERENCIAS}

Engel-Cox, J.A., Holloman, C.H., Coutant, B.W., Hoff, R.M. 2004. Qualitative and quantitative evaluation of MODIS satellite sensor data for regional and urban scale air quality. Atmospheric Environment, 38: 2495-2509.

Evans, J., van Donkelaar, A., Martin, R.V., Burnett, R., Rainham, D.G., Birkett, N.J., Krewski, D. 2013. Estimates of global mortality attributable to particulate air pollution using satellite imagery. Environmental Research, 120: 33-42.

Gupta, P., Christopher, S.A., Wang, J., Gehrig, R., Lee, Yc., Kumar, N. 2006. Satellite remote sensing of particulate matter and air quality assessment over global cities. Atmospheric Environment, 40: 5880-5892.

$\mathrm{Hu}$, Z. 2009. Spatial analysis of MODIS aerosol optical depth, $\mathrm{PM}_{2,5}$, and chronic coronary heart disease. International Journal of Health Geographics, 8:27.

Hutchison, S.S., Smith, S., Faruqui, S. 2004. The use of MODIS data and aerosol products for air quality prediction. Atmospheric Environment, 38: 5057-5070.

Kim, K.H., Kabir, E., Kabir, S. 2015. A review on the human health impact of airborne particulate matter. Environmental International, 74: 136-143.

Katz, R. 2006. Contaminación del aire en Santiago: estamos mejor que lo creemos pero a mitad de camino. En Galetovic, A. (Ed.). Santiago. Dónde Estamos y Hacia Dónde Vamos. Santiago: Centro de Estudios Públicos (CEP), pp. 332-369. 
Kumar, N., Chu, A., Foster, A. 2007. An empirical relationship between $\mathrm{PM}_{2,5}$ and aerosol optical depth in Delhi Metropolitan. Atmospheric Environment, 41: 4492-4503.

Liang, S., Zhong, B., Fang, H. 2006. Improved estimation of aerosol optical depth from MODIS imagery over land surfaces. Remote Sensing of Environment, 104(4): 416-425.

Liu, Y.J., Franklin, M., Kahn, R., Koutrakis, P. 2007. Using aerosol optical thickness to predict groundlevel $\mathrm{PM}_{2,5}$ concentrations in the St. Louis area: A comparison between MISR and MODIS. Remote Sensing of Environment, 107: 33-44.

Liu, Y., Sarnat, J.A., Kilaru, V., Jacob, D.J., Koutrakis, P. 2005. Estimating ground-level $P_{2,5}$ in the Eastern United States using satellite remote sensing. Environmental Science and Technology, 39: 3269-3278.

Paciorek, C.J., Liu, Y. 2009. Limitations of remotely sensed aerosol as a spatial proxy for fine particulate matter. Environmental Health Perspectives, 117(6): 904-909.

SEGPRES (Ministerio Secretaría General de la Presidencia de Chile). 2010. Decreto 66. SINCA [en línea] [fecha de consulta: Julio de 2016] http://sinca.mma.gob.cl/uploads/documentos/2011 0105 DTO-66 16-ABR-2010 4546.pdf

Smith, P., Romero, H. 2016. Factores explicativos de la distribución espacial de la temperatura del aire de verano en Santiago. Revista Geográfica Norte Grande, 63: 45-62.

Tsai, T-C., Jeng, Y-J., Chue, D.A., Chen, J-P., Chang, S-C. 2011. Analysis of the relationship between MODIS aerosol optical depth and particulate matter from 2006 to 2008. Atmospheric Environment, 45: 4777-4788.

Van Donkelaar, A., Martin, R.V., Brauer, M., Kahn, R., Levy, R., Verduzco, C., Villenueve, P.J. (2010). Global estimates of ambient fine particulate matter concentrations from satellite-based aerosol optical depth: Development and application. Environmental Health Perspectives, 118(6): 847855.
Van Donkelaar, A., Martin, R.V., Park, R.J. (2006) Estimating ground-level $\mathrm{PM}_{2,5}$ using aerosol optical depth determined from satellite remote sensing. Journal of Geophysical Research, 111: D21201.

Wang, J., Christopher, S.A. (2003). Intercomparison between satellite-derived aerosol optical thickness and $\mathrm{PM}_{2,5}$ mass: Implications for air quality studies. Geophysical Research Letters, 30(21): 4-1-4-4.

Willumsen, H. (2010). El Estado de la Calidad de Aire en Chile. CONAMA [en línea] [fecha de consulta: Junio de 2016] http://sinca.mma.gob.cl/uploads/documentos/23b2f 3aeedcb0ca6f5815b62ac6bc414.pdf 